\title{
NONSMOOTH STABILIZABILITY AND FEEDBACK LINEARIZATION OF DISCRETE-TIME NONLINEAR SYSTEMS
}

\author{
C. SIMÕES AND H. NIJMEUER \\ University of Twente, Department of Applied Mathematics, PO Box 217, 7500 AE Enschede, The Netherlands \\ AND \\ J. TSINIAS* \\ National Technical University, Department of Mathematics, Zografou Campus, 15780 Athens, Greece
}

\begin{abstract}
SUMMARY
We consider the problem of stabilizing a discrete-time nonlinear system using a feedback which is not necessarily smooth. A sufficient condition for global dynamical stabilizability of single-input triangular systems is given. We obtain conditions expressed in terms of distributions for the nonsmooth feedback triangularization and linearization of discrete-time systems. Relations between stabilization and linearization of discrete-time systems are given.
\end{abstract}

KEY WORDS nonlinear discrete-time systems; nonsmooth stabilization; feedback linearization

\section{INTRODUCTION}

In this paper we consider the problems of stabilizing and of linearizing a single-input discretetime nonlinear control system of the form

$$
x(k+1)=f(x(k), u(k))
$$

where $x \in \mathbb{R}^{n}$ and $u \in \mathbb{R}$, using coordinate transformations and state feedbacks which are not necessarily smooth.

The smooth stabilization problem for (1) consists of finding a smooth feedback law $u=u(x)$ which renders the closed-loop system asymptotically stable for some equilibrium point $\bar{x}$. Some results regarding the stabilizability of the system (1) have been reported in Reference 9. In the present note we want to contribute further to the question of designing a stabilizing controller for (1) that renders the equilibrium $\bar{x}$ asymptotically stable. Essentially the innovative element of this paper lies in two points, namely we admit for controllers that are implicitly defined as a dynamic controller and secondly we do not necessarily require our feedback to possess smoothness properties. It is interesting to note that this work has some points in common with implicit control equations as used in differential algebraic control (see Reference 4) and the topological methods used in continuous-time nonlinear systems by Celikovský in Reference 2 .

\footnotetext{
-This work was performed while this author was visiting the University of Twente as a Research Fellow.

This paper was recommended for publication by editor A. Isidori

CCC 1049-8923/96/030171-18

(C) 1996 by John Wiley \& Sons, Ltd.

Received 11 February 1994

Revised 27 June 1994
} 
In Section 2, after introducing a natural notion of dynamical asymptotic stability, we obtain a sufficient condition for an asymptotically stabilizable single-input discrete-time control system to be dynamically asymptotically stabilizable. This result (Lemma 2.3) will be useful in Section 3 , when dealing with the stabilization of triangular systems. The continuous-time version of Lemma 2.3 received already some attention, see for instance Reference 3.

In Section 3 we study the problem of dynamic asymptotic stabilization for triangular systems with a nonsmooth feedback. Some sufficient conditions are given for systems with state space $\mathbb{R}^{n}$. These conditions are analogous with those given in Reference 11 for stabilization of continuous-time triangular systems. Note at this point that the methodology employed in the present paper for stabilization of the discrete-time case is less technical and does not consider a discrete analogous procedure of Reference 11. Finally, two examples are presented, where for given nonlinear systems stabilizing explicit feedback control laws are constructed.

The smooth feedback linearization problem for the system (1) consists of finding a smooth coordinate change $z=\phi(x)$ and a smooth feedback $u=\alpha(x, v)$ such that in the new coordinates the system becomes a linear controllable one. This problem has been studied and solved locally by Grizzle ${ }^{5}$ and Jakubczyk. ${ }^{6}$ Reference 1 , a survey of results and methods on stability of continuous-time systems, presents feedback linearization as an indirect method for stabilization. See also Reference 8 for a self-contained presentation of the results. In Section 4 we study feedback equivalence of discrete-time systems using nonsmooth transformations: for that, we will give conditions for the triangularization of a system and conditions for a triangular system to be linearizable. Notice that for stabilizability of triangular systems we have the results obtained in Section 3; connecting then both Sections 3 and 4 we obtain results on the stability of the original system and both its triangularization and its linearization. The connection between stabilization and feedback linearization has already been suggested in Reference 2 for continuous-time nonlinear systems, where the author uses the notion of feedback linearization to approach the stabilization of smoothly nonstabilizable nonlinear systems.

\section{DEFINITIONS AND PRELIMINARY RESULTS}

Consider the implicit discrete-time autonomous system

$$
\Sigma: F(x(k), x(k+1))=0
$$

where $F: \mathbb{R}^{2 n} \rightarrow \mathbb{R}^{p}$ for some natural $p$, is a continuous map such that $F(0,0)=0$. The family of systems of the form (2) includes all the difference equations in explicit form

$$
x(k+1)=f(x(k))
$$

but also systems of this form on which some restrictions are imposed, for instance, the state space being a subset of $\mathbb{R}^{n}$ of the form $\{x \mid g(x)=0\}$. Since in the sequel we will include systems with this kind of restriction, in this section we will give the terminology according to the more general structure (2).

Let $\mathbb{N}$ be the set of natural numbers, $\mathbb{N}_{0}=\mathbb{N} \cup\{0\}$ and $\mathscr{P}\left(\mathbb{R}^{n}\right)$ the set of all subsets of $\mathbb{R}^{n}$. Given a system $\Sigma$ of the form (2), define the map $A_{\Sigma}: \mathbb{N}_{o} \times \mathbb{R}^{n} \rightarrow \mathscr{P}\left(\mathbb{R}^{n}\right)$ where

$$
\begin{array}{r}
A_{\Sigma}\left(k, x_{0}\right)=\left\{x \in \mathbb{R}^{n} \mid \exists x_{1}, \ldots, x_{k-1} \in \mathbb{R}^{n}: F\left(x_{o}, x_{1}\right)=0,\right. \\
\left.\quad F\left(x_{1}, x_{2}\right)=0, \ldots, F\left(x_{k-2}, x_{k-1}\right)=0, F\left(x_{k-1}, x\right)=0\right\}
\end{array}
$$

for $\left(k, x_{0}\right) \in \mathbb{N} \times \mathbb{R}^{n}$ and $A_{\Sigma}\left(0, x_{0}\right)=\left\{x_{0}\right\}$. We call $A_{\Sigma}\left(k, x_{o}\right)$ the solution set of $\Sigma$ at time $k$ starting from $x_{v}$. The system $\Sigma$ is called complete if for any $x \in \mathbb{R}^{n}$ there exist $y \in \mathbb{R}^{n}$ such that 
$F(x, y)=0$. Completeness of $\Sigma$ is equivalent to the fact that $A_{\Sigma}\left(k, x_{o}\right) \neq Q$, for all $x_{o} \in \mathbb{R}^{n}$ and $k \in \mathbb{N}_{o}$. Obviously, if $\Sigma$ has the form (3), then it is complete and for every $k$ and $x_{o}$ the set $A_{\Sigma}\left(k, x_{o}\right)$ is a singleton.

We say that $0 \in \mathbb{R}^{n}$ is an equilibrium point for $\Sigma$ if $A_{\Sigma}(1,0)=\{0\}$.

\section{Definition 2.1}

Let $\Sigma$ be a system of the form (2), with dynamics $F$ vanishing at zero and $0 \in \mathbb{R}^{n}$ an equilibrium point for $\Sigma$.

(a) We say that $0 \in \mathbb{R}^{n}$ is locally asymptotically stable (L.A.S.) with respect to $\Sigma$ if

(i) the system $\Sigma$ is complete;

(ii) for any $\varepsilon>0$, there exist $\delta=\delta(\varepsilon)>0$ such that if $\left\|x_{o}\right\|<\delta$, then $\|y\|<\varepsilon$, $\forall y \in A_{\Sigma}\left(k, x_{o}\right), \forall k \in \mathbb{N}_{o}$;

(iii) there exist $\delta>0$ such that if $\left\|x_{o}\right\|<\delta$ then $\lim _{k \rightarrow \infty} y(k)=0$, where $y(k)$ is any in $A_{\Sigma}\left(k, x_{o}\right)$, for each $k \in \mathbb{N}_{o}$.

We say that $0 \in \mathbb{R}^{n}$ is globally asymptotically stable (G.A.S.) with respect to $\Sigma$ if it is L.A.S. and furthermore $\lim _{k \rightarrow \infty} y(k)=0$, where $y(k)$ is any in $A_{\Sigma}\left(k, x_{o}\right)$, for each $k \in \mathbb{N}_{o}$ and every initial $x_{v} \in \mathbb{R}^{n}$.

(b) $\Sigma$ has the global (resp. local) dead beat property of order $k_{o}\left(k_{o} \in \mathbb{N}\right)$ if $0 \in \mathbb{R}^{n}$ is G.A.S. (resp. L.A.S.) and $A_{\Sigma}\left(k, x_{o}\right)=\{0\}, \forall k>k_{o}, \forall x_{o} \in \mathbb{R}^{n}$ (resp. $\forall x_{o} \in \mathbb{R}^{n}$ such that $\left\|x_{o}\right\|<\delta$, with $\delta$ defined as in (a)(iii)).

Consider now the single-input discrete-time control system

$$
x(k+1)=f(x(k), u(k))
$$

where $f: \mathbb{R}^{n+1} \rightarrow \mathbb{R}^{n}$ is a continuous map with $f(0,0)=0$. Applying the static feedback $u=u(x)$ in (4) we obtain a system of the form (3). If, more generally, the feedback law verifies a dynamic implicit equation as $\varphi(x(k), u(k), u(k+1))=0$, we obtain a system of the form (2), with state given by $\left[x^{\mathrm{T}} u\right]^{\mathrm{T}}$. In that case the corresponding dynamic feedback is called implicit dynamic compensator of order one. Using the notion of implicit dynamic compensator, we introduce the following definition.

\section{Definition 2.2}

(a) The system (4) is globally (resp. locally) dynamically asymptotically stabilizable (G.D.A.S.) (resp. L.D.A.S.) at $0 \in \mathbb{R}^{n}$ if there exists a $C^{0}$ map $\varphi: \mathbb{R}^{n+2} \rightarrow \mathbb{R}$ such that $0 \in \mathbb{R}^{n+1}$ is G.A.S. (resp. L.A.S.) with respect to the closed-loop implicit system

$$
\left\{\begin{array}{l}
x(k+1)-f(x(k), u(k))=0 \\
\varphi(x(k), u(k), u(k+1))=0
\end{array}\right.
$$

(b) The system (4) has the global (resp. local) dynamic dead beat property of order $k_{o}$ $\left(k_{o} \in \mathbb{N}\right.$ ) with respect to $\varphi$ if in addition (5) has the global (resp. local) dead beat property of order $k_{0}$.

Notice that the origin $0 \in \mathbb{R}^{n+1}$ is an equilibrium point for (5) if and only if the map $\varphi$ satisfies

$$
\varphi(0,0, u)=0 \Leftrightarrow u=0
$$

The following lemma gives a sufficient condition for (4) to be G.D.A.S. (resp. L.D.A.S.). 


\section{Lemma 2.3}

Consider the control system (4).

(a) Suppose that there exist a $C^{0}$ map $\psi: \mathbb{R}^{n+1} \rightarrow \mathbb{R}$ satisfying $\psi(0,0)=0$ and

$$
\left[\lim _{k \rightarrow \infty} \psi(x(k), \mu(k))=0 \wedge \lim _{k \rightarrow \infty} x(k)=0\right] \Rightarrow\left[\lim _{k \rightarrow \infty} u(k)=0\right]
$$

and such that $0 \in \mathbb{R}^{n}$ is G.A.S. (resp. L.A.S.) with respect to the system

$$
\Sigma_{1}:\left\{\begin{array}{l}
x(k+1)-f(x(k), u(k))=0 \\
\psi(x(k), u(k))=0
\end{array}\right.
$$

Then the system (4) is G.D.A.S. (resp. L.D.A.S.) by means of the dynamic compensator

$$
\varphi(x(k), u(k), u(k+1))=\psi(f(x(k), u(k)), u(k+1))
$$

(b) Suppose that $\Sigma_{1}$ has the dead beat property of order $k_{r}$. Then the system (4) has the dynamic dead beat property of order $k_{o}+1$ with respect to $\varphi$.

Notice that the state for the system $\Sigma_{1}$ is $x$ : in fact, given the equation $\psi(x(k), u(k))=0$ in $\mathbb{R}^{n+1}$ and given a state $x(k)$ of $\Sigma_{1}$ at time $k$, we get the admissible $u(k)$ s which, plugged into the first equation $x(k+1)-f(x(k), u(k))=0$, allow us to obtain $A_{\Sigma_{1}}(1, x(k))$. For this reason is $u(k)$ in $\Sigma_{1}$ just a latent variable and we can consider this system as being of the form (2).

Proof. We prove the lemma only for the global case. The local result follows analogously.

(a) Our first claim is to prove that $0 \in \mathbb{R}^{n+1}$ is G.A.S. with respect to the system

$$
\Sigma_{2}:\left\{\begin{array}{l}
x(k+1)-f(x(k), u(k))=0 \\
\psi(f(x(k), u(k)), u(k+1))=0
\end{array}\right.
$$

Let $\{(x(k), u(k))\}$ be any sequence of vectors such that $(x(k), u(k)) \in A_{\Sigma_{2}}\left(k,\left(x_{o}, u_{o}\right)\right)$, for each $k \in \mathbb{N}_{o}$. Then we have

$$
\begin{aligned}
& x(1)=f\left(x_{o}, u_{o}\right) \\
& \psi\left(f\left(x_{o}, u_{o}\right), u(1)\right)=0 \Leftrightarrow \psi(x(1), u(1))=0 \\
& x(2)=f(x(1), u(1)) \\
& \psi(f(x(1), u(1)), u(2))=0 \Leftrightarrow \psi(x(2), u(2))=0 \\
& \vdots \\
& x(k)=f(x(k-1), u(k-1)) \\
& \psi(f(x(k-1), u(k-1)), u(k))=0 \Leftrightarrow \psi(x(k), u(k))=0 \\
& \vdots
\end{aligned}
$$

Therefore the sequence $\{(x(k), u(k))\}$ also satisfies, for each $k \in \mathbb{N}$, the pair of equations

$$
\left\{\begin{array}{l}
x(k+1)=f(x(k), u(k)) \\
\psi(x(k), u(k))=0
\end{array}\right.
$$

with $x(1)=f\left(x_{o}, u_{o}\right)$ and $u(1)$ being any scalar such that $\psi(x(1), u(1))=0$. It follows that the system $\Sigma_{2}$ is also complete and for all $k \in \mathbb{N}$

$$
(x(k), u(k)) \in A_{\Sigma_{1}}(k-1, x(1)) \times\{u(k)\}
$$

Since $x(k) \in A_{\Sigma_{1}}(k-1, x(1))$, we have $\lim _{k \rightarrow \infty} x(k)=0$. Since $\psi$ verifies (6), then we also have $\lim _{k \rightarrow \infty} u(k)=0$. 
Now from the fact that for all $k \in \mathbb{N}$

$$
A_{\Sigma_{2}}\left(k+1,\left(x_{o}, u_{o}\right)\right) \subset A_{\Sigma_{1}}(k, x(1)) \times\{u(k+1)\}
$$

we conclude that $0 \in \mathbb{R}^{n+1}$ is G.A.S. with respect to $\Sigma_{2}$.

(b) Our second claim is to prove that

$$
A_{\Sigma_{2}}\left(k,\left(x_{o}, u_{o}\right)\right)=\{0\}, \quad \forall k \geqslant k_{o}+1, \quad \forall\left(x_{o}, u_{o}\right) \in \mathbb{R}^{n+1}
$$

Since $\psi$ is continuous and verifies (6), we also have $(\psi(0, u)=0 \Rightarrow u=0)$.

The result now follows from (8).

The continuous-time version of the result presented in Lemma 2.3 , namely whether a system $\dot{x}=f(x, y)$ is dynamically asymptotically stabilizable by means of a feedback integrator has been explored in References 3, 10 and 11. In particular, in Reference 3 this problem has been solved for a wide class of systems whereas the corresponding dynamic feedback is smooth for $(x, u) \neq 0$ and continuous at zero. In References 10 and 11 necessary and sufficient conditions of Lyapunov type are presented for the existence of a nonsmooth feedback integrator. These conditions are analogous to those imposed in Lemma 2.3, however as we have mentioned in the introduction, the methodology is quite different from the one in the present paper.

\section{Remark 2.4}

A particular case arises when the equation $\psi(x, u)=0$ is solvable with respect to $u$, namely that there exist a unique (continuous) map $u=U(x)$ such that $\psi(x, U(x))=0, \forall x$, which means that this map makes zero G.A.S. with respect to the system (4). In that case, the map $\varphi$ defined in (7) is also solvable with respect to the last entry, i.e.,

$$
\varphi(x, u, \hat{u})=0 \Leftrightarrow \psi(f(x, u), \hat{u})=0 \Leftrightarrow \hat{u}=U(f(x, u))
$$

therefore $\Sigma_{2}$ can be written in an explicit form as

$$
\Sigma_{2}:\left\{\begin{array}{l}
x(k+1)=f(x(k), u(k)) \\
u(k+1)=U(f(x(k), u(k))
\end{array}\right.
$$

and Lemma 2.3 is a generalization of Proposition 3.2 in Reference 9 for the single-input case.

\section{SYSTEMS HAVING A TRIANGULAR STRUCTURE}

In this section we will consider the single-input discrete-time nonlinear control system having the following triangular structure

$$
\left\{\begin{array}{l}
x_{1}(k+1)=f_{1}\left(x_{1}(k), x_{2}(k)\right) \\
x_{2}(k+1)=f_{2}\left(x_{1}(k), x_{2}(k), x_{3}(k)\right) \\
\vdots \\
x_{n-1}(k+1)=f_{n-1}\left(x_{1}(k), x_{2}(k), \ldots, x_{n}(k)\right) \\
x_{n 3}(k+1)=f_{n}\left(x_{1}(k), x_{2}(k), \ldots, x_{n}(k), x_{n+1}(k)\right) \\
x_{n+1}=u
\end{array}\right.
$$

where $f_{i}: \mathbb{R}^{i+1} \rightarrow \mathbb{R}, i=1,2, \ldots, n$, are continuous functions vanishing at zero and $\left(x_{1}, \ldots, x_{n}\right) \in \mathbb{R}^{n}$ and $u \in \mathbb{R}$ are the state and the input of the system (9), respectively. Let us make the following assumption for (9). 
Assumption 3.1

(a) There exists a $C^{0}$ map $x_{2}=X_{2}\left(x_{1}\right)$ vanishing at zero which globally asymptotically stabilizes the system

$$
x_{1}(k+1)=f_{1}\left(x_{1}(k), x_{2}(k)\right)
$$

at zero, i.e., $0 \in \mathbb{R}$ is G.A.S. with respect to the system

$$
x_{1}(k+1)=f_{1}\left(x_{1}(k), X_{2}\left(x_{1}(k)\right)\right)
$$

(b) For every $i=2, \ldots, n$ and for each $x_{1}, \ldots, x_{i}$, the map $f_{i}\left(x_{1}, \ldots, x_{i}, \cdot\right)$ is a surjection.

(c) For every $i=1, \ldots, n$, the following holds

$$
\left.\begin{array}{r}
\lim _{k \rightarrow \infty} f_{i}\left(x_{1}(k), \ldots, x_{i}(k), x_{i+1}(k)\right)=0 \\
\lim _{k \rightarrow \infty}\left(x_{1}(k), \ldots, x_{i}(k)\right)=0
\end{array}\right\} \Rightarrow \lim _{k \rightarrow \infty} x_{i+1}(k)=0
$$

The next theorem shows that Assumption 3.1 is a sufficient condition for constructing a global stabilizing feedback for the system (9).

\section{Theorem 3.2}

If Assumption 3.1 holds then the system (9) is G.D.A.S. at zero. If we further assume that (11) has the dead beat property of order $k_{o}$ then (9) has the dynamic dead beat property of order $k_{o}+n$ with respect to some map $\varphi: \mathbb{R}^{n+2} \rightarrow \mathbb{R}$.

Proof. We prove by induction that for every $p=1, \ldots, n$, there exists a continuous map $\varphi_{p}: \mathbb{R}^{p+2} \rightarrow \mathbb{R}$ vanishing at zero such that $0 \in \mathbb{R}^{p+1}$ is G.A.S. with respect to the system

$$
\left\{\begin{array}{l}
x_{1}(k+1)=f_{1}\left(x_{1}(k), x_{2}(k)\right) \\
x_{2}(k+1)=f_{2}\left(x_{1}(k), x_{2}(k), x_{3}(k)\right) \\
\vdots \\
x_{p}(k+1)=f_{p}\left(x_{1}(k), x_{2}(k), \ldots, x_{p+1}(k)\right) \\
\varphi_{p}\left(x_{1}(k), \ldots, x_{p+1}(k), x_{p+1}(k+1)\right)=0
\end{array}\right.
$$

For the case $p=1$, consider the system $x_{1}(k+1)=f_{1}\left(x_{1}(k), x_{2}(k)\right)$ and define

$$
\psi_{1}\left(x_{1}, x_{2}\right)=x_{2}-X_{2}\left(x_{1}\right)
$$

where $X_{2}$ is the map defined in Assumption 3.1(a). By Assumption 3.1(a), $0 \in \mathbb{R}$ is G.A.S. with respect to

$$
\left\{\begin{array}{l}
x_{1}(k+1)=f_{1}\left(x_{1}(k), x_{2}(k)\right) \\
\psi_{1}\left(x_{1}(k), x_{2}(k)\right)=0
\end{array}\right.
$$

On the other hand, (13) satisfies the assumptions of Lemma 2.3, therefore $0 \in \mathbb{R}^{2}$ is G.A.S. with respect to the system

$$
\left\{\begin{array}{l}
x_{1}(k+1)=f_{1}\left(x_{1}(k), x_{2}(k)\right) \\
\varphi_{1}\left(x_{1}(k), x_{2}(k), x_{2}(k+1)\right)=0
\end{array}\right.
$$


where

$$
\varphi_{1}\left(x_{1}, x_{2}, \hat{x}_{2}\right)=\psi_{1}\left(f_{1}\left(x_{1}, x_{2}\right), \hat{x}_{2}\right)
$$

Suppose now that our claim holds for $p<n$, namely that there exists a $C^{0}$ map $\varphi_{p}: \mathbb{R}^{p+2} \rightarrow \mathbb{R}$ vanishing at zero and such that

(i) the following property holds

$$
\left.\begin{array}{r}
\lim _{k \rightarrow \infty} \varphi_{p}\left(x_{1}(k), \ldots, x_{p+1}(k), \hat{x}_{p+1}(k)\right)=0 \\
\lim _{k \rightarrow \infty}\left(x_{1}(k), \ldots, x_{p+1}(k)\right)=0
\end{array}\right\} \Rightarrow \lim _{k \rightarrow \infty} \hat{x}_{p+1}(k)=0
$$

(ii) for every $x_{1}, \ldots, x_{p+1}$, the equation

$$
\varphi_{p}\left(x_{1}, \ldots, x_{p+1}, \hat{x}_{p+1}\right)=0
$$

is solvable (not necessarily uniquely) with respect to the variable $\hat{x}_{p+1}$ (which implies the completeness of (12)),

(iii) the origin $0 \in \mathbb{R}^{p+1}$ is G.A.S. with respect to (12).

Consider now the $(p+1)$ th equation of $(9)$

$$
x_{p+1}(k+1)=f_{p+1}\left(x_{1}(k), \ldots, x_{p+1}(k), x_{p+2}(k)\right)
$$

Since $f_{p+1}$ verifies Assumption 3.1(c), from the induction hypothesis follows that $0 \in \mathbb{R}^{p+1}$ is also G.A.S. with respect to

$$
\left\{\begin{array}{l}
x_{1}(k+1)=f_{1}\left(x_{1}(k), x_{2}(k)\right) \\
x_{2}(k+1)=f_{2}\left(x_{1}(k), x_{2}(k), x_{3}(k)\right) \\
\vdots \\
x_{p}(k+1)=f_{p}\left(x_{1}(k), x_{2}(k), \ldots, x_{p+1}(k)\right) \\
x_{p+1}(k+1)=f_{p+1}\left(x_{1}(k), x_{2}(k), \ldots, x_{p+1}(k), x_{p+2}(k)\right) \\
\varphi_{p}\left(x_{1}(k), \ldots, x_{p+1}(k), f_{p+1}\left(x_{1}(k), \ldots, x_{p+1}(k), x_{p+2}(k)\right)\right)=0
\end{array}\right.
$$

Conditions (i) and (ii) above together with Assumption 3.1(b) and (c), imply that for any $x_{1}, \ldots, x_{p+1}$, the equation

with

$$
\psi_{p+1}\left(x_{1}, \ldots, x_{p+1}, x_{p+2}\right)=0
$$

$$
\psi_{p+1}\left(x_{1}, \ldots, x_{p+1}, x_{p+2}\right)=\varphi_{p}\left(x_{1}, \ldots, x_{p+1}, f_{p+1}\left(x_{1}, \ldots, x_{p+1}, x_{p+2}\right)\right)
$$

is solvable with respect to $x_{p+2}$, which implies completeness of (14) and furthermore the following holds

$$
\left.\begin{array}{r}
\lim _{k \rightarrow \infty} \psi_{p+1}\left(x_{1}(k), \ldots, x_{p+1}(k), x_{p+2}(k)\right)=0 \\
\lim _{k \rightarrow \infty}\left(x_{1}(k), \ldots, x_{p+1}(k)\right)=0
\end{array}\right\} \Rightarrow \lim _{k \rightarrow \infty} x_{p+2}(k)=0
$$

The previous discussion guarantees that the system (14) satisfies the conditions of Lemma 2.3 with

$$
f=\left[\begin{array}{c}
f_{1} \\
\vdots \\
f_{p+1}
\end{array}\right], \quad \psi=\psi_{p+1}, \quad x=\left[\begin{array}{c}
x_{1} \\
\vdots \\
x_{p+1}
\end{array}\right]
$$


and so $0 \in \mathbb{R}^{p+2}$ is G.A.S. with respect to the system

$$
\left\{\begin{array}{l}
x_{1}(k+1)=f_{1}\left(x_{1}(k), x_{2}(k)\right) \\
x_{2}(k+1)=f_{2}\left(x_{1}(k), x_{2}(k), x_{3}(k)\right) \\
\vdots \\
x_{p+1}(k+1)=f_{p+1}\left(x_{1}(k), x_{2}(k), \ldots, x_{p+1}(k), x_{p+2}(k)\right) \\
\psi_{p+1}\left(f_{1}\left(x_{1}(k), x_{2}(k)\right), \ldots, f_{p+1}\left(x_{1}(k), \ldots, x_{p+1}(k), x_{p+2}(k)\right), x_{p+2}(k+1)\right)=0
\end{array}\right.
$$

Then our claim also holds for $p+1$, with

$$
\varphi_{p+1}\left(x_{1}, \ldots, x_{p+2}, \hat{x}_{p+2}\right)=\psi_{p+1}\left(f_{1}\left(x_{1}, x_{2}\right), \ldots, f_{p+1}\left(x_{1}, \ldots, x_{p+2}\right), \hat{x}_{p+2}\right)
$$

Note finally that using the previous properties of $\varphi_{p}$ we can easily establish that both conditions (i) and (ii) hold for the map $\varphi_{p+1}$. For reasons of completeness, we prove (ii), namely we show that the equation

$$
\varphi_{p+1}\left(x_{1}, \ldots, x_{p+2}, \hat{x}_{p+2}\right)=0
$$

is solvable with respect to $\hat{x}_{p+2}$. Indeed, by Assumption $3.1(\mathrm{~b})$ it follows that for every $x_{1}, \ldots, x_{p+2}$ the equation

$$
\varphi_{p}\left(f_{1}\left(x_{1}, x_{2}\right), \ldots, f_{p+1}\left(x_{1}, \ldots, x_{p+2}\right), v\right)=0
$$

is solvable on $v$. For the same reason, for every $x_{1}, \ldots, x_{p+2}$ and $v$ as above, the equation

$$
v=f_{p+1}\left(f_{1}\left(x_{1}, x_{2}\right), \ldots, f_{p+1}\left(x_{1}, \ldots, x_{p+1}, x_{p+2}\right), \hat{x}_{p+2}\right)
$$

is solvable with respect to $\hat{x}_{p+2}$. The desired conclusion follows then by the definitions (15) and (16).

The dead beat property follows similarly.

A local version of Theorem 3.2 can be given as follows.

\section{Corollary 3.3}

Suppose that for the system (9)

(a) There exists a $C^{0}$ map $x_{2}=X_{2}\left(x_{1}\right)$ vanishing at zero which locally asymptotically stabilizes the system (10) at zero.

(b) For each $i=2, \ldots, n$, there is an open interval $I_{i} \subset \mathbb{R}$ containing zero such that $f_{i}\left(x_{1}, \ldots, x_{i}, I_{i}\right)$ is again an open interval containing zero, for each $x_{1}, \ldots, x_{i}$.

(c) For every $i=1, \ldots, n$,

$$
\left.\begin{array}{rl}
\lim _{k \rightarrow \infty} & f_{i}\left(x_{1}(k), \ldots, x_{i}(k), x_{i+1}(k)\right)=0 \\
\lim _{k \rightarrow \infty}\left(x_{1}(k), \ldots, x_{i}(k)\right) & =0
\end{array}\right\} \Rightarrow \lim _{k \rightarrow \infty} x_{i+1}(k)=0
$$

Then the system (9) is L.D.A.S. at zero.

\section{Remark 3.4}

If we assume in Assumption 3.1(b) that

(i) also $f_{1}\left(x_{1}, \cdot\right)$ is surjective, for each $x_{1}$,

(ii) $\mathscr{S}=\left\{\left(x_{1}, x_{2}\right) \in \mathbb{R}^{2} \mid f_{1}\left(x_{1}, x_{2}\right)=0\right\}$ is a connected set,

then Assumption 3.1 (a) is automatically true. 
In fact, in that case there exists a continuous real function $X_{2}\left(x_{1}\right)$ defined for all $x_{1} \in \mathbb{R}$, vanishing at zero and such that

$$
f_{1}\left(x_{1}, X_{2}\left(x_{1}\right)\right)=0, \quad \forall x_{1} \in \mathbb{R}
$$

The function $X_{2}\left(x_{1}\right)$, defined for all initial $\bar{x}_{1}(0)$, can be taken as the map referred to in Assumption 3.1 (a) and is constructed in such a way that the system

$$
x_{1}(k+1)=f_{1}\left(x_{1}(k), X_{2}\left(x_{1}(k)\right)\right)
$$

has the dead beat property of order 1 .

Now from Theorem 3.2 we can conclude that the system (9) has the dynamic dead beat property of order $n+1$ (with respect to the function $\varphi$ referred to in Theorem 3.2).

We illustrate the above comments with some examples. The first one is a discrete-time counterpart of the planar nonsmooth stabilization as described in Reference 7.

\section{Example 3.5}

Consider the following system of the form (9):

$$
\left\{\begin{array}{l}
x_{1}(k+1)=-3 x_{1}(k)+x_{2}^{3}(k) \\
x_{2}(k+1)=u^{3}(k)
\end{array}\right.
$$

In this case, we have

$$
\left\{\begin{array}{l}
f_{1}\left(x_{1}, x_{2}\right)=-3 x_{1}+x_{2}^{3} \\
f_{2}\left(x_{1}, x_{2}, u\right)=u^{3}
\end{array}\right.
$$

Since $f_{1}\left(x_{1}, \cdot\right)$ and $f_{2}\left(x_{1}, x_{2}, \cdot\right)$ are surjections and the set

$$
\mathscr{S}=\left\{\left(x_{1}, x_{2}\right) \mid-3 x_{1}+x_{2}^{3}=0\right\}
$$

is connected, the system (17) is G.D.A.S. To determine a stabilizing feedback law, define as

$$
X_{2}\left(x_{1}\right)=\left(3 x_{1}\right)^{1 / 3}
$$

the map referred to in Assumption 3.1(a). Now using Lemma 2.3, $0 \in \mathbb{R}^{2}$ is G.A.S. with respect to the system

$$
\left\{\begin{array}{l}
x_{1}(k+1)=-3 x_{1}(k)+x_{2}^{3}(k) \\
x_{2}(k+1)=\left[-9 x_{1}(k)+3 x_{2}^{3}(k)\right]^{1 / 3}
\end{array}\right.
$$

Then a stabilizing feedback law for the system (17) is

$$
u\left(x_{1}, x_{2}\right)=\left[-9 x_{1}+3 x_{2}^{3}\right]^{1 / 9}
$$

which is in fact a $C^{0}$-function. On the other hand, the linearization of (17) is

$$
z(k+1)=\left[\begin{array}{rr}
-3 & 0 \\
0 & 0
\end{array}\right] z(k)+\left[\begin{array}{l}
0 \\
0
\end{array}\right] v(k)
$$

which is uncontrollable and unstable. 
It is known that if a system is stabilizable by use of a $C^{\prime}$ feedback law then the uncontrollable modes of the linearization are stable. Example 3.5 does not contradict this result, since the constructed feedback law is not $C^{1}$. Nevertheless, we should not believe that it is always possible to construct a $C^{0}$ feedback to stabilize a system whose linearization has unstable uncontrollable modes; for instance, in Reference 9 it is shown that the system $x(k+1)=f(x(k))+B u(k)$ is stabilizable by a feedback for which no continuity assumption is imposed only if the uncontrollable modes of the linearization are stable.

The next example shows that the arguments used in Example 3.5 can be used for systems with higher dimension, contrary to the continuous-time case presented in Reference 7 which does not seem to hold in dimension larger than 2.

\section{Example 3.6}

Consider the following system of the form (9)

$$
\left\{\begin{array}{l}
x_{1}(k+1)=2 x_{1}(k)+x_{2}^{p}(k) \\
x_{2}(k+1)=x_{3}^{q}(k) \\
x_{3}(k+1)=u^{r}(k)
\end{array}\right.
$$

where $p, q, r$ are odd numbers. In this case, we have

$$
\left\{\begin{array}{l}
f_{1}\left(x_{1}, x_{2}\right)=2 x_{1}+x_{2}^{p} \\
f_{2}\left(x_{1}, x_{2}, x_{3}\right)=x_{3}^{q} \\
f_{3}\left(x_{1}, x_{2}, x_{3}, u\right)=u^{r}
\end{array}\right.
$$

Since $f_{1}\left(x_{1}, \cdot\right), f_{2}\left(x_{1}, x_{2}, \cdot\right)$ and $f_{3}\left(x_{1}, x_{2}, x_{3}, \cdot\right)$ are surjections and

$$
\mathscr{P}=\left\{\left(x_{1}, x_{2}\right) \mid 2 x_{1}+x_{2}^{p}=0\right\}
$$

is connected, the system (18) is G.D.A.S. To determine a stabilizing feedback law, define as

$$
X_{2}\left(x_{1}\right)=-\left(2 x_{1}\right)^{1 / p}
$$

the map referred to in Assumption 3.1 (a). Define

$$
\psi_{1}\left(x_{1}, x_{2}\right)=x_{2}-X_{2}\left(x_{1}\right)
$$

Using Lemma $2.3,0 \in \mathbb{R}^{2}$ is G.A.S. with respect to the system

$$
\left\{\begin{array}{l}
x_{1}(k+1)=2 x_{1}(k)+x_{2}^{p}(k) \\
x_{2}(k+1)=-\left[4 x_{1}(k)+2 x_{2}^{p}(k)\right]^{1 / p}
\end{array}\right.
$$

Now $x_{2}(k+1)=x_{3}^{4}(k)$, so using again Lemma $2.3,0 \in \mathbb{R}^{3}$ is G.A.S. with respect to

$$
\left\{\begin{array}{l}
x_{1}(k+1)=2 x_{1}(k)+x_{2}^{p}(k) \\
x_{2}(k+1)=x_{3}^{q}(k) \\
x_{3}(k+1)=-\left[8 x_{1}(k)+4 x_{2}^{p}(k)+2 x_{3}^{p q}(k)\right]^{1 / p q}
\end{array}\right.
$$


Then a stabilizing feedback law for the system (18) is

$$
u\left(x_{1}, x_{2}, x_{3}\right)=-\left[8 x_{1}+4 x_{2}^{p}+2 x_{3}^{p q}\right]^{1 / p q r}
$$

which is again a $C^{0}$ function.

Notice that again the linearization of the system (18) is uncontrollable and unstable.

Both feedback laws obtained in the previous examples are continuous, but this was not guaranteed by Theorem 3.2. (In fact, they are both almost smooth, i.e., globally continuous and smooth except at a set $\mathscr{S}$ of measure zero: in case of Example 3.5 the curve

$$
\mathscr{S}=\left\{\left(x_{1}, x_{2}\right) \in \mathbb{R}^{2}:-9 x_{1}+3 x_{2}^{3}=0\right\}
$$

and in case of Example 3.6 the surface

$$
\left.\mathscr{S}=\left\{\left(x_{1}, x_{2}, x_{3}\right) \in \mathbb{R}^{3}: 8 x_{1}+4 x_{2}^{p}+2 x_{3}^{\mathrm{pq}}=0\right\}\right)
$$

The reason for this is that the functions $f_{1}\left(x_{1}, \cdot\right), f_{2}\left(x_{1}, x_{2}, \cdot\right)$ and $f_{3}\left(x_{1}, x_{2}, x_{3}, \cdot\right)$ are in fact bijections, and this is a sufficient condition for finding a continuous stabilizing feedback law for (9), as we will see in the next corollary.

We now strengthen Assumption 3.1 as follows.

\section{Assumption 3.7}

(a) The same as Assumption 3.1 (a).

(b) For each $i=2, \ldots, n$ and for every $x_{1}, \ldots, x_{i}$, the map $f_{i}\left(x_{1}, x_{2}, \ldots, x_{i}, \cdot\right)$ is bijective.

(c) The same as Assumption 3.1 (c).

\section{Corollary 3.8}

If Assumption 3.7 holds, then (9) is G.D.A.S. by means of an almost smooth feedback.

Proof. The proof follows the same arguments as those of the proof of Theorem 3.2. In addition, take into account Remark 2.4 and the fact that, because of the Assumption 3.7, for each $p+1=1, \ldots, n$, the map $\psi_{p+1}$ as defined in (15) is uniquely solvable with respect to $x_{p+2}$. In particular, there exists a $C^{0}$ map $X_{p+2}: \mathbb{R}^{p+1} \rightarrow \mathbb{R}$ such that

$$
\psi_{p+1}\left(x_{1}, \ldots, x_{p+1}, X_{p+2}\left(x_{1}, \ldots, x_{p+1}\right)\right)=0, \forall x_{1}, \ldots, x_{p+1}
$$

Let now $x_{n+1}=\varphi_{\mathrm{c}}(x)$, with $x=\left(x_{1}, \ldots, x_{n}\right)$, be a continuous global stabilizer for (9). By the well-known converse Lyapunov theorem for discrete-time systems there exists a continuous positive definite and uniformly unbounded function $V: \mathbb{R}^{n} \rightarrow \mathbb{R}^{+}$such that

$$
V\left(f_{1}\left(x_{1}, x_{2}\right), \ldots, f_{n}\left(x_{1}, \ldots, x_{n}, \varphi_{\mathrm{c}}(x)\right)\right)<V(x), \quad \forall x \neq 0
$$

The previous inequality asserts that for every nonzero $x$ we have

$$
V\left(f_{1}\left(x_{1}, x_{2}\right), \ldots, f_{n}\left(x_{1}, \ldots, x_{n}, v\right)\right)<V(x)
$$

for every $v$ in an open neighbourhood $\mathcal{N}_{x}$ of the vector $\varphi_{c}(x)$.

Define $U=\cup_{x \neq 0} \mathcal{N}_{x}$. Then $U$ is a covering of the graph of the map $\varphi_{c}(x), x \neq 0$. Using (20) and standard partition of unity arguments (see Reference 9) we can now construct an almost smooth map $x_{n+1}=\varphi(x), \varphi(0)=0$, whose graph is contained in $u$ and such that (19) is satisfied with $\varphi$ instead of $\varphi_{c}$. This implies that $\varphi$ globally asymptotically stabilizes (9). 


\section{NONSMOOTH FEEDBACK LINEARIZATION OF DISCRETE-TIME NONLINEAR SYSTEMS}

The goal of feedback linearization consists in finding conditions for the existence of a feedback law $u=u(x, v)$ for which a given system is transformed into a linear one, possibly after a smooth change of coordinates in the state space.

The purpose of this section is to study the feedback linearization of discrete-time systems using transformations which are not necessarily smooth and characterize those systems which are feedback equivalent to a linear one. In fact, we will deal with coordinate changes and feedbacks that are almost everywhere analytic or, more precisely, that are analytic on an open and dense part of the space where they are defined. For instance, the feedbacks obtained in Examples 3.5 and 3.6 are both analytic except respectively at a curve in $\mathbb{R}^{2}$ and at a surface in $\mathbb{R}^{3}$.

Consider the single-input discrete-time control system

$$
x(k+1)=f(x(k), u(k))
$$

where $x \in \mathbb{R}^{n}, u \in \mathbb{R}$ and $f: \mathbb{R}^{n+1} \rightarrow \mathbb{R}^{n}$ is an analytic map such that $f(0,0)=0$.

We define the following (nonsmooth) transformations for (21):

- A local regular static state feedback for (21) on a given open subset $\mathscr{X}$ of $\mathbb{R}^{n}$ is a relation $u=\alpha(x, v)$ for which there exists an open interval $V \subset \mathbb{R}$ such that the map $\alpha(x, \cdot): \mathcal{V} \rightarrow \alpha(x, \eta) \subset \mathbb{R}$ is bijective and continuous, $\forall x \in \mathscr{X}$.

- A local coordinate change of (21) around a given $x_{o} \in \mathbb{R}^{n}$ is a bijective and continuous map $\mathscr{Y}: \mathscr{Q} \subset \mathbb{R}^{n} \rightarrow \mathscr{S}(\mathscr{X}) \subset \mathbb{R}^{n}$ defined on a neighbourhood $\mathscr{X}$ of $x_{o}$.

In this section we are interested in finding conditions under which (21) can be put into the form (9) by means of a coordinate change and/or a regular static state feedback. When that is possible, we say that (21) and (9) are locally feedback equivalent or that (21) is locally feedback triangularizable.

More precisely, we introduce the following.

\section{Definition 4.1}

Consider the system (21) and a point $\left(x_{o}, u_{o}\right) \in \mathbb{R}^{n+1}$. The system (21) is locally feedback equivalent to the system

around $\left(x_{o}, u_{o}\right)$ if there exist

$$
z(k+1)=g(z(k), v(k))
$$

(a) a coordinate transformation $\mathscr{Y}: \mathscr{X} \subset \mathbb{R}^{n} \rightarrow \mathscr{Y}(\mathscr{X}) \subset \mathbb{R}^{n}$ defined on a neighbourhood $\mathscr{X}$ of $x_{o}$ with $\mathscr{Y}\left(x_{0}\right)=z_{o}$,

(b) a regular static state feedback $u=\alpha(x, v)$ defined on a neighbourhood $\mathscr{X} \times \mathscr{V}$ of $\left(x_{0}, v_{o}\right)$ and satisfying $\alpha\left(x_{o}, v_{o}\right)=u_{o}$

such that in the new coordinates $z=\mathscr{S}(x)$ the closed-loop dynamics are given by (22) in a neighbourhood of $\left(z_{o}, v_{o}\right)$.

Of course the most common situation is the case where the system (22) is linear. In that case, the system (21) is said to be locally feedback linearizable around $\left(x_{o}, u_{o}\right)$.

The following proposition gives sufficient conditions for the system (9) to be feedback linearizable. 


\section{Proposition 4.2}

Consider the system (9) and suppose that for a given $\left(x_{o}, u_{0}\right)$ with $x_{o} \equiv\left(x_{o 1}, \ldots, x_{o n}\right)$ there are open intervals $I_{1}, \ldots, I_{n}$ containing respectively $x_{o 2}, \ldots, x_{o n}, u_{o}$ such that the functions

$$
f_{i}\left(x_{1}, \ldots, x_{i}, \cdot\right): I_{i} \rightarrow f_{i}\left(x_{1}, \ldots, x_{i}, I_{i}\right)
$$

are bijective, for every $x_{1}, \ldots, x_{i}, i=1, \ldots, n$.

Then (9) is locally feedback equivalent to a controllable linear system around $\left(x_{o}, u_{o}\right)$.

Proof. In the system (9) $f$ has the following structure

$$
f(x, u)=\left[\begin{array}{l}
f_{1}\left(x_{1}, x_{2}\right) \\
f_{2}\left(x_{1}, x_{2}, x_{3}\right) \\
\vdots \\
f_{n-1}\left(x_{1}, x_{2}, \ldots, x_{n}\right) \\
f_{n}\left(x_{1}, \ldots, x_{n}, u\right)
\end{array}\right]
$$

Choose around $\left(x_{o}, u_{o}\right)$ new coordinates $\left(z_{1}, \ldots, z_{n}\right)$ as follows:

$$
\left\{\begin{array}{l}
z_{1}=x_{1} \\
z_{2}=f_{1}\left(x_{1}, x_{2}\right) \\
z_{k}=x_{k}, k=3, \ldots, n
\end{array}\right.
$$

Then $x_{2}=f_{1}^{*}\left(x_{1}, x_{2}\right)$ for some function $f_{1}^{*}$ with $f_{1}^{*}\left(x_{1}, \cdot\right)$ bijective in some interval containing $f_{1}\left(x_{1}, x_{o 2}\right)$, and the map $f$ will take the form:

$$
\tilde{f(z, u)}=\left[\begin{array}{l}
z_{2} \\
\overline{f_{2}}\left(z_{1}, z_{2}, z_{3}\right) \\
\vdots \\
\overline{f_{n}}\left(z_{1}, \ldots, z_{n}, u\right)
\end{array}\right]
$$

with $\overline{f_{2}}, \ldots, \overline{f_{n}}$ bijective in the last entry, in some open intervals. If we repeat the process for $\overline{f_{2}}, \ldots, \overline{f_{n}}$, we will get

$$
\tilde{f(z, u)}=\left[\begin{array}{l}
z_{2} \\
\vdots \\
z_{n} \\
\tilde{f_{n}}(z, u)
\end{array}\right]
$$

where $\tilde{f}_{n}(z, \cdot)$ is bijective around $u_{0}$. Take now the regular static state feedback $v=\tilde{f}_{n}(z, u)$. In the new coordinates and around $\left(x_{o}, u_{o}\right)$, we obtain the system

$$
z(k+1)=\left[\begin{array}{l}
z_{2}(k) \\
\vdots \\
z_{n}(k) \\
v(k)
\end{array}\right]
$$

which is a controllable linear system. 
Obviously, if $I_{i}=\mathbb{R}, i=1, \ldots, n$, then (9) is globally feedback linearizable around any point $(x, u) \in \mathbb{R}^{n+1}$.

We now return to (21) to find conditions under which this system is feedback equivalent to a system of the form (9). Denote by $\pi$ the canonical projection $\pi(x, u)=x$ and by $\mathscr{K}$ the distribution $\mathcal{K}=\operatorname{ker} f_{*}$ defined in $U \subset \mathbb{R}^{n+1}$, where $\mathcal{U}$ is the set of points where $f_{*}$ has full rank, with $f_{*}$ the Jacobian of $f$. Since $f$ is analytic, if $f_{*}$ has full rank at some point of $\mathbb{R}^{n+1}$ then $f_{*}$ has full rank in an open and dense part of $\mathbb{R}^{n+1}$. From this we can conclude that if $u$ is nonempty, then it is open and dense in $\mathbb{R}^{n+1}$.

We associate with the system (21) the following sequence of distributions (Reference 8, pp. 439-440).

\section{Algorithm 4.3}

Consider the set $\mathcal{U} \subset \mathbb{R}^{n+1}$ where $f *$ has full rank.

Step 0. For $(x, u) \in \mathcal{U}$, define the distribution

$$
D_{0}=\pi_{*}^{-1}(0)
$$

Step $i+1$. Consider the set $u_{i} \subset \mathbb{R}^{n+1}$ where $D_{i}+\mathscr{K}$ is an involutive constant-dimensional distribution. $u_{i}$ is open and dense in $\mathbb{R}^{n+1}$. Define for $(x, u) \in \boldsymbol{U}_{i}$ the distribution

$$
D_{i+1}=\pi_{*}^{-1} f_{*}\left(D_{i}\right)
$$

and stop if $D_{i+1}+\mathscr{K}$ is not involutive or constant-dimensional in an open and dense part of $\mathbb{P}^{n+1}$ or if $\operatorname{dim} D_{i+1}=n+1$.

We illustrate this algorithm with an example.

\section{Example 4.4}

Consider the system (17) from Example 3.5. Then

$$
f\left(x_{1}, x_{2}, u\right)=\left(-3 x_{1}+x_{2}^{3}, u^{3}\right)
$$

and

$$
f_{*}\left(x_{1}, x_{2}, u\right)=\left[\begin{array}{ccc}
-3 & 3 x_{2}^{2} & 0 \\
0 & 0 & 3 u^{2}
\end{array}\right]
$$

$f_{*}$ has full rank except if $u=0$, therefore $u=\left\{\left(x_{1}, x_{2}, u\right): u \neq 0\right\}$. For $(x, u) \in U$, define

$$
\mathscr{K}\left(x_{1}, x_{2}, u\right)=\operatorname{ker} f_{*}\left(x_{1}, x_{2}, u\right)=\operatorname{span}\left\{\left[\begin{array}{c}
x_{2}^{2} \\
1 \\
0
\end{array}\right]\right\}
$$

Then $\operatorname{dim} \mathscr{H}(x, u)=1$, for all $(x, u) \in \mathcal{u}$. For $(x, u) \in u$, define

$$
D_{0}(x, u)=\pi_{*}^{-1}(0)(x, u)=\operatorname{span}\left(\left[\begin{array}{l}
0 \\
0 \\
1
\end{array}\right]\right)
$$


$\left(D_{0}+\mathscr{K}\right)(x, u)$ is involutive and constant-dimensional for $(x, u) \in \mathcal{U}$, so $\boldsymbol{u}_{0}=\mathcal{U}$. For $(x, u) \in u_{0}$, define

$$
D_{1}\left(x_{1}, x_{2}, u\right)=\pi_{*}^{-1} f_{*}\left(D_{0}\right)\left(x_{1}, x_{2}, u\right)=\operatorname{span}\left(\left[\begin{array}{c}
0 \\
u^{2} \\
0
\end{array}\right],\left[\begin{array}{l}
0 \\
0 \\
1
\end{array}\right]\right)
$$

Then $\left(D_{1}+\mathscr{K}\right)(x, u)$ is involutive and constant-dimensional for $(x, u) \in \mathcal{U}_{1}=$ $\left\{\left(x_{1}, x_{2}, u\right): u \cdot x_{2} \neq 0\right\}$. For $(x, u) \in \mathcal{U}_{1}$, let

$$
D_{2}\left(x_{1}, x_{2}, u\right)=\pi_{*}^{-1} f_{*}\left(D_{1}\right)\left(x_{1}, x_{2}, u\right)=\operatorname{span}\left(\left[\begin{array}{c}
x_{2}^{2} u^{2} \\
0 \\
0
\end{array}\right],\left[\begin{array}{c}
0 \\
u^{2} \\
0
\end{array}\right],\left[\begin{array}{l}
0 \\
0 \\
1
\end{array}\right]\right)
$$

Then $\left(D_{2}+\mathscr{K}\right)(x, u)$ is involutive and of constant dimension for $(x, u) \in \boldsymbol{U}_{2}=\boldsymbol{U}_{1}$ and $\operatorname{dim} D_{2}(x, u)=3, \forall(x, u) \in \mathcal{U}_{2}$.

Using Algorithm 4.3 we obtained distributions $D_{0}, D_{1}, D_{2}$ which are all involutive and constant dimensional in $\varkappa_{2}$, an open and dense subset of $\mathbb{R}^{3}$.

In Proposition 4.2 we obtained sufficient conditions for a triangular system to be feedback linearizable. The next step is to obtain conditions for the system (21) to be feedback equivalent to a triangular one. Those conditions are presented in the Theorems 4.5 (sufficient conditions) and 4.7 (necessary conditions).

\section{Theorem 4.5}

Consider the discrete-time nonlinear system (21). If Algorithm 4.3 applied to (21) gives distributions $D_{0}, \ldots, D_{n}$ defined respectively in $\varkappa_{0}, \ldots, \varkappa_{n}$ and such that

$$
\operatorname{dim} D_{i}(x, u)=i+1,(x, u) \in u_{i}, \quad i=0, \ldots, n
$$

then (21) is on an open dense subset of $\mathbb{R}^{n}$ feedback equivalent to a system of the form (9). The obtained triangular system is such that in each connected component of $\boldsymbol{u}_{n}$ all functions $f_{i}\left(x_{1}, \ldots, x_{i}, \cdot\right)$ are injective, for each $x_{1}, \ldots, x_{i}, i=1, \ldots, n$.

Proof. Suppose that $\operatorname{dim} D_{i}(x, u)=i+1,(x, u) \in \mathcal{u}_{i}, i=0, \ldots, n$. According to Algorithm 4.3, we have $u_{1} \supset u_{2} \supset \ldots \supset u_{n}$. Given an arbitrary point $\left(x_{o}, u_{o}\right) \in u_{n}$, let $\bar{u}_{n}$ be the connected component of $u_{n}$ which contains $\left(x_{u}, u_{o}\right)$. For $(x, u) \in \bar{u}_{n}$ define

$$
\Delta_{i}(x, u)=\pi_{*} D_{i}(x, u), \quad i=1, \ldots, n
$$

Since $\operatorname{dim} D_{i}=i+1$, we have $\operatorname{dim} \Delta_{i}=i$. Then, as in Reference 8 (p. 442), it is possible to obtain local coordinates $x$ in $\bar{u}_{n}$ such that

$$
\Delta_{i}=\operatorname{span}\left\{\frac{\partial}{\partial x_{n-i+1}}, \ldots, \frac{\partial}{\partial x_{n}}\right\}, i=1, \ldots, n
$$

With respect to the above coordinates $x$, let $f_{i}$ be the $i$-component of $f$, for $i=1, \ldots, n$. In a similar way as in Reference 8 (pp. 442-443), we investigate the structure of $f$ with respect to 
the distributions $\Delta_{i}, i=1, \ldots, n$. From (24) and (25), we have that $f_{*} D_{0}=\Delta_{1}$, which implies that in $\bar{u}_{n}$

$$
\operatorname{span}\left\{\sum_{j=1}^{n} \frac{\partial f_{j}}{\partial u}(x, u) \frac{\partial}{\partial x_{j}}\right\}=\operatorname{span}\left\{\frac{\partial}{\partial x_{n}}\right\}
$$

yielding

$$
\frac{\partial f_{j}}{\partial u}(x, u)=0, \text { for } j=1, \ldots, n-1 \text { and also } \frac{\partial f_{n}}{\partial u}(x, u) \neq 0
$$

Similarly, from $f_{*} D_{1}=\Delta_{2}$, we have

$$
\operatorname{span}\left\{\sum_{j=1}^{n} \frac{\partial f_{j}}{\partial x_{n}}(x, u) \frac{\partial}{\partial x_{j}}, \frac{\partial f_{n}}{\partial u}(x, u) \frac{\partial}{\partial x_{n}}\right\}=\operatorname{span}\left\{\frac{\partial}{\partial x_{n-1}}, \frac{\partial}{\partial x_{n}}\right\}
$$

from which we obtain

$$
\frac{\partial f_{j}}{\partial x_{n}}(x, u)=0, \text { for } j=1, \ldots, n-2 \text { and } \frac{\partial f_{n-1}}{\partial x_{n}}(x, u) \neq 0
$$

A repetition of the above argument using $f_{*} D_{i-1}=\Delta_{i}, i=1, \ldots, n$, yields the triangular form (23) for $f$, with

$$
\frac{\partial f_{i-1}}{\partial x_{i}}(x, u) \neq 0, i=1, \ldots, n+1\left(x_{n+1}=u\right)
$$

for each $(x, u) \in \bar{u}_{n}$. Since $\left(x_{o}, u_{o}\right)$ is arbitrary, we can conclude that in each connected component of $u_{n}$ the functions $f_{i}\left(x_{1}, \ldots, x_{i}, \cdot\right)$ are injective, for each $x_{1}, \ldots, x_{i}$, for $i=1, \ldots, n$.

If additionally the $D_{i} \mathrm{~s}$ can be defined everywhere, we obtain a global result. More precisely, we obtain the following.

\section{Corollary 4.6}

Under the conditions of Theorem 4.5, suppose that there exists involutive and constantdimensional distributions $\bar{D}_{0}, \ldots, \bar{D}_{n}$ in $\mathbb{R}^{n+1}$ such that

$$
\bar{D}_{i}(x, u)=D_{i}(x, u),(x, u) \in u_{i}, \quad i=1, \ldots, n
$$

Then (21) is globally feedback equivalent to a system of the form (9).

\section{Theorem 4.7}

Suppose that

- the system (21) is feedback equivalent to a triangular system $\Sigma_{\mathrm{T}}$ of the form (9) in an open and dense part $\ell$ of $\mathbb{R}^{n+1}$.

- given any connected component $\bar{u}$ of $u, \bar{u}$ is a convex set and the functions $f_{i}$ in $\Sigma_{\mathrm{T}}$ are such that

$$
f_{i}\left(x_{1}, \ldots, x_{i}, \cdot\right): \bar{u}_{i}\left(x_{1}, \ldots, x_{i}\right) \rightarrow \mathbb{R}
$$


is injective, for any $x_{1}, \ldots, x_{i}, i=1, \ldots, n$, where

$$
\bar{u}_{i}\left(x_{1}, \ldots, x_{i}\right)=\left\{x \in \mathbb{R}:\left(x_{1}, \ldots, x_{i}, x_{i+2}, \ldots, x_{n}\right) \in \bar{u} \text {, for some } x_{i+2}, \ldots, x_{n} \in \mathbb{R}\right\}
$$

Then Algorithm 4.3 applied to the system (21) gives distributions $D_{0}, \ldots, D_{n}$ which are involutive and constant dimensional on $u$ and such that $\operatorname{dim} D_{i}=i+1, i=0, \ldots, n$.

Proof. Let $\bar{u}$ be a connected component of $\boldsymbol{U}$. In $\overline{\boldsymbol{U}},(21)$ is feedback equivalent to a triangular system $\Sigma_{\mathrm{T}}$ of the form (9), with $f$ given by (23) and $f_{i}\left(x_{1}, \ldots, x_{i}, \cdot\right)$ injective when restricted to $\bar{u}_{i}\left(x_{1}, \ldots, x_{i}\right)$, for $i=1, \ldots, n$.

Since $\vec{u}$ is convex, $\vec{u}_{i}$ is a connected set and the map

$$
f_{i}\left(x_{1}, \ldots, x_{i}, \cdot\right): \bar{\varphi}_{i} \rightarrow f_{i}\left(x_{1}, \ldots, x_{i}, \bar{\varphi}_{i}\right)
$$

is bijective, for each $i=1, \ldots, n$.

Using now Proposition 4.2 on the system $\Sigma_{\mathrm{T}}$ with $f: \bar{u} \rightarrow f(\bar{U})$, we conclude that (21) is feedback equivalent in $\bar{u}$ to a controllable linear system

$$
z(k+1)=A z(k)+b v(k)
$$

In these coordinates, $f_{*}=[A \mid b]$, which has full rank since $(26)$ is controllable. Now the claim follows from the fact that

$$
\begin{aligned}
& D_{0}=\operatorname{span}\left\{\frac{\partial}{\partial v}\right\} \\
& \vdots \\
& D_{i}=\operatorname{Imb}+\cdots+A^{i-1} \operatorname{Imb}+\operatorname{span}\left\{\frac{\partial}{\partial v}\right\}
\end{aligned}
$$

Combining respectively the results of Corollary 3.8 with those in Proposition 4.2 and Theorem 4.5 we easily obtain the following two corollaries.

\section{Corollary 4.8}

Suppose that for the system (9) $f_{i}\left(x_{1}, \ldots, x_{i}, \cdot\right)$ is bijective, for each $x_{1}, \ldots, x_{i}, i=1, \ldots, n$. Then the system (9) is G.D.A.S. at $0 \in \mathbb{R}^{n}$ and globally feedback linearizable around any point $(x, u) \in \mathbb{R}^{n+1}$.

\section{Corollary 4.9}

Consider the discrete-time nonlinear system (21). If

- the Algorithm 4.3 applied to (21) gives distributions $D_{0}, \ldots, D_{n}$ defined respectively in $u_{0}, \ldots, u_{n}$ and such that

$$
\operatorname{dim} D_{i}(x, u)=i+1,(x, u) \in \mathfrak{U}_{i}, \quad i=0, \ldots, n,
$$

- the origin is contained in $u_{n}$,

- the resulting triangular system verifies Assumption 3.1(c),

then there is a neighbourhood of the origin contained in $\boldsymbol{U}_{n}$ where the triangular system is L.D.A.S. 


\section{CONCLUSIONS}

In this paper we have addressed the stabilization problem and the feedback linearization problem for discrete-time nonlinear systems. For the special case of triangular systems, we obtained sufficient conditions for both its stabilization and linearization. We gave some examples to illustrate how to construct a stabilizing feedback for an $n$-dimensional triangular system. Furthermore, we proposed sufficient conditions for a triangular system to be linearizable and for a general system to be triangularizable. Finally, relations between stabilization and linearization of discrete-time nonlinear systems have been explored.

\section{ACKNOWLEDGEMENT}

The work of C.S., on leave from the University of Coimbra, Portugal, was supported by the Junta Nacional de Investigação Científica e Tecnológica under Grant BD/1214/91-RM.

\section{REFERENCES}

1. Baccionti, A., Local Stabilizability of Nonlinear Control Systems, World Scientific, Singapore, 1992.

2. Celikovský, S., 'Topological linearization of nonlinear systems: application to the nonsmooth stabilization', in Proceedings of the ECC 93, Groningen, 1993, pp. 41-44.

3. Coron, J. M. and L. Praly, 'Adding an integrator for the stabilization problem', Systems and Control Letters, 17, 89-104 (1991).

4. Fliess, M., 'Generalized controller canonical forms for linear and nonlinear dynamics', IEEE Trans. Automatic Control, AC-35, 994-1001 (1990).

5. Grizzle, J. W., 'Feedback linearization of discrete-time systems', in Analysis and Optimization of Systems, A. Bensoussan and J. L. Lions (Eds), Lecture Notes Control Information Science, Vol, 83, Springer-Verlag, Berlin, 1986, pp. 273-284.

6. Jakubczyk, B., 'Feedback linearization of discrete-time systems', Systems and Control Letters, 9, 411-416 (1987).

7. Kawski, M., 'Stabilization of nonlinear systems in the plane', Systems and Control Letters, 12, 169-175 (1989).

8. Nijmeijer, H. and A. J. van der Schaft, Nonlinear Dynamical Control Systems, Springer Verlag, New York, 1990.

9. Tsinias, J., 'Stabilizability of discrete-time nonlinear systems', IMA Journal of Mathematical Control and Information, 6, 135-150 (1989).

10. Tsinias, J., 'An extension of Artstein's theorem on stabilization by using ordinary feedback integrator', Systems and Control Letters, 20, 141-148 (1993).

11. Tsinias, J., 'Smoothly global stabilizability by dynamic feedback and generalizations of Arstein's theorem', in Proceedings of the Int. Conference on Control Theory and Appl., Israel, 1993. 